\title{
Quadratic Phase Matching in slot waveguides
}

\author{
Andrea Di Falco \\ NooEL - Nonlinear Optics and OptoElectronics Lab, University "Roma Tre"- Rome, Italy \\ Claudio Conti \\ Research Center "Enrico Fermi", Via Panisperna 89/A - Rome, Italy \\ Research Center Soft INFM-CNR, University "La Sapienza", Piazzale A. Moro, 2 - 00185 - Rome, Italy
}

\section{Gaetano Assanto}

NooEL - Nonlinear Optics and OptoElectronics Lab

Department of Electronic Engineering, INFN and CNISM, University "Roma Tre"

Via della Vasca Navale, 84 - 00146 - Rome, Italy

Compiled June 26, 2021

\begin{abstract}
We analyze phase matching with reference to frequency doubling in nanosized quadratic waveguides encompassing form birefringence and supporting cross-polarized fundamental and second harmonic modes. In an AlGaAs rod with an air-void, we show that phase-matched second-harmonic generation could be achieved in a wide spectral range employing state-of-the-art nanotechnology. (c) 2021 Optical Society of America

OCIS codes: 190.4360, 190.4390
\end{abstract}

Since the pioneering work by Franken et al.,$^{1}$ frequency doubling has been among the most studied nonlinear processes in optics. Conversion efficiency in second harmonic generation (SHG) relates to spatio-temporal overlap of waves at fundamental (FF) and second harmonic (SH) frequencies, as well as to their group and phase velocity matching. For the latter, in order to compensate material and waveguide dispersion, several approaches have been proposed and demonstrated, ${ }^{2}$ including birefringent phase-matching $(\mathrm{PM})$, non-critical PM, quasi$\mathrm{PM}(\mathrm{QPM})^{3}$ in co- or counter-propagating geometries, including vertical QPM for surface-emitted SHG. ${ }^{4-7}$ More recently, novel PM schemes for SHG include random quasi-PM ${ }^{8}$ photonic crystals and microcavities, ${ }^{9,}, 10$ as well as form-birefringence in waveguides. ${ }^{11-14}$ Formbirefringence conjugates the large intensities and interaction distances of guided-waves with the possibility of tailoring the dispersion of cross-polarized waves even in isotropic crystals such as GaAs and its composites. ${ }^{12-14}$ It consists in alternating layers with different refractive indices orthogonally to the direction of propagation, such that the harmonics experience distinct eigen-values for TE (quasi-TE) and TM (quasi-TM) polarizations. As in photonic crystals, the degree of birefringence, its dispersion and the achievable PM line-width are directly linked to the index contrast between alternating films and their thicknesses ${ }^{15}$ making film growth a formidable task. ${ }^{14}$ For these reasons, a nano-sized structure encompassing form-birefringence but realizable with just one nonlinear material should provide significant advantages and versatility over standard approaches for parametric mixing and frequency generation.

In this Letter we propose and investigate SHG in a suspended rod-waveguide with a nanometric void, imple- menting form-birefringence and large modal overlap for efficient upconversion. To achieve the highest index contrast between a quadratically nonlinear semiconductor (AlGaAs) and air (or vacuum), we consider the basic geometry sketched in Fig. [1 with the electric field of the FF mode parallel to the void (i.e., quasi-TM with respect to the top surface) and a cross-polarized (i.e., quasi-TE) SH tightly confined in the air-gap owing to the discontinuity in the electric field normal to the interfaces, the latter separated by $v$. Fig. 团also displays the calculated FF and $\mathrm{SH}$ lowest-order eigen-profiles of such a slot waveguide, similar to the one recently studied by $\mathrm{Xu}$ et al. for ringresonators in $\mathrm{SiO}_{2} .{ }^{16}$ We consider cubic $\mathrm{Al}_{0.3} \mathrm{Ga}_{0.7} \mathrm{As}$ with indices $n_{F F}$ and $n_{S H}$ at $\mathrm{FF}$ and $\mathrm{SH}$, respectively, after Ref. [17]. The insertion of a symmetrically-placed air-gap of width $v$ allows to phase-match the quadratic interaction between a quasi- $T M_{00}$ mode of wavelength $\lambda_{F F}$ and its quasi- $T E_{00}$ second harmonic, leaving nearly unaltered the effective second-order susceptibility.

To explore PM in the structure, we fixed the slot-

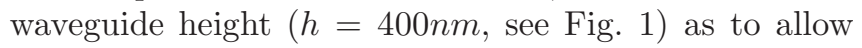
single-mode confinement at $\mathrm{FF}$ and form-birefringence PM for SHG over the explored spectrum. Variations in $h$ modify the pertinent propagation constants and the PM wavelengths, but only marginally affect the modal overlap integrals and the conversion.

We calculated the effective indices versus slot aperture $v$ and individual rod-width $w$ resorting to a finitedifference semi-vectorial mode-solver. This was validated against a (computationally heavy) fully-vectorial solver and yielded accurate results for the nanometric geometry under investigation. We also exploited symmetry and solved the eigenvalue problem for a quarter of the (transverse) geometry. 


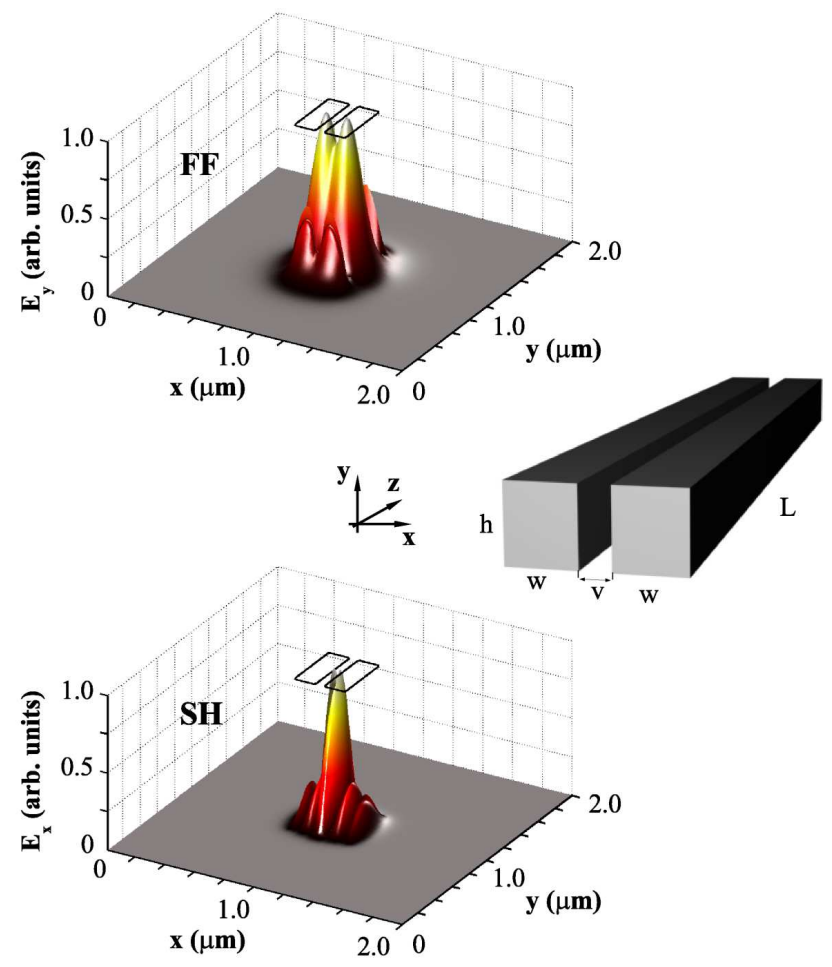

Fig. 1. Sketch of the air-void waveguide and corresponding lowest-order 3D eigen-profiles. Top: quasi-TM FF; bottom: quasi-TE SH. Here $h=400 \mathrm{~nm}, w=150 \mathrm{~nm}$, $v=50 \mathrm{~nm}$ and $\lambda=1550 \mathrm{~nm}$ at FF.

Fig. 22 shows the computed effective indices $\beta \lambda / 2 \pi$ for FF (thick lines) and $\mathrm{SH}$ (thin lines) versus $v$ and for three widths $w$ at $\lambda_{F F}=1550 \mathrm{~nm}$. The curves at $\lambda_{S H}=\lambda_{F F} / 2$ exhibit a much stronger dispersion due to the filling-factor of the eigensolution in the gap: the $\mathrm{SH}$ mode is much more affected by small changes in $v$ than the FF, the latter exhibiting a nearly linear dispersion. Clearly, this becomes more appreciable as $v$ approaches zero, whereas dispersion flattens for large $v$.

Fig. 3 (top) graphs a set of pairs $(v, w)$ for which $\Delta \beta=\beta\left(\lambda_{S H}\right)-2 \beta\left(\lambda_{F F}\right)=0$, i.e., type I phase matching. For each pair we computed the modal overlap integral and the quadratic susceptibility $\Gamma=$ $\iint_{S} d(x, y)\left(E_{y, F F}\right)^{2} E_{x, S H} d x d y$, with $S$ the cross section and the fields normalized as to yield the modal power $P=\iint|E(x, y)|^{2} d x d y . d(x, y)$ is the spatial distribution of the susceptibility coupling a $\hat{y}$-polarized FF to an $\hat{x}$ polarized SH through the element $d_{14}$. In our waveguide, $d(x, y)=d_{14}$ within the $w \times h$ rods - cut on a (001) substrate after a 45 rotation with respect to crystal axes ${ }^{14}$ - and $d(x, y)=0$ elsewhere. As an increase in $w$ forces the mode to fill more the nonlinear dielectric, but the higher $w$ the higher is the required $v$ to achieve PM, $\Gamma$ exhibits a maximum. The corresponding parameters yield the highest conversion efficiency per given propagation length.

The dependence of SHG efficiency on $\lambda_{F F}$ is graphed in Fig. 团along with the phase mismatch for a slot waveg-

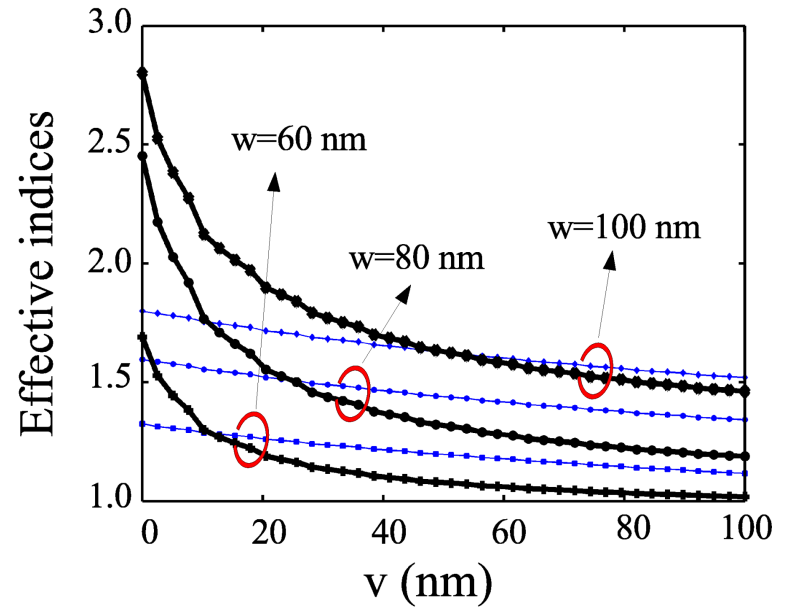

Fig. 2. Effective indices at FF (thick lines) and SH (thin lines) vs void $v$ for $\lambda_{F F}=1550 \mathrm{~nm}$ and three values of $w$. Crossings of paired curves correspond to type I PM for SHG.

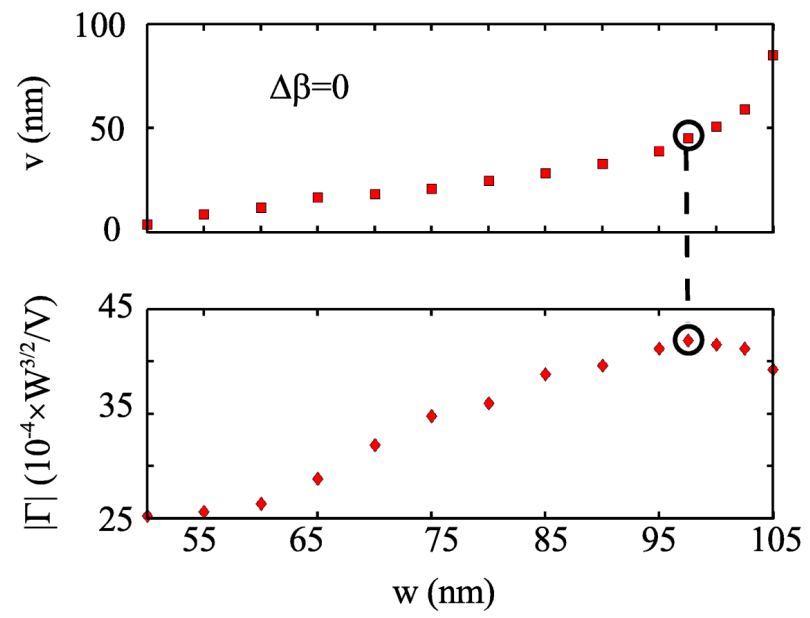

Fig. 3. Top: PM condition $(\Delta \beta=0)$ versus $v$ and $w$ (in $n m$ ); bottom: corresponding moduli of the SHG overlap integral. Circles indicate the values providing maximum conversion efficiency.

uide of unity length $L=1 \mathrm{~mm}$, accounting for dispersion. Suspended membranes of lengths and aspect-ratios comparable to the one proposed here have been recently realized. ${ }^{18,19}$ The top panel shows conversion $\eta$ and $\Delta \beta L$ versus $\lambda_{F F}$ for the optimum parameters encircled in Fig. 3 and unity $(1 W)$ excitation, corresponding to a (material) power density of $\approx 400 \mathrm{MW} / \mathrm{cm}^{2}$ and comparable to what previously employed for SHG in GaAs/AlGaAs guides. ${ }^{13}$ Clearly, a large SHG is associated with the peak of a sinc-like curve and a specific PM condition (horizontal dashes) near $\lambda_{F F}=1.55 \mu \mathrm{m}$. Such an individual zero for $\Delta \beta$, however, is not the norm. As displayed in the bottom of Fig. 3, a slight detuning from the optimum $v$ shifts and splits the PM-wavelength, giving rise to a double SH resonance with a mismatch remaining moder- 
ate between the two zeroes. This stems from the strong birefringence of the structure, yielding different dispersion for the two cross-polarized harmonics. For a given void, while TM-like modes substantially undergo material dispersion, TE $\mathrm{SH}$ is quite sensitive to wavelength changes, resulting in a multi-peaked SHG conversion efficiency amenable to engineering towards broad-band doublers.
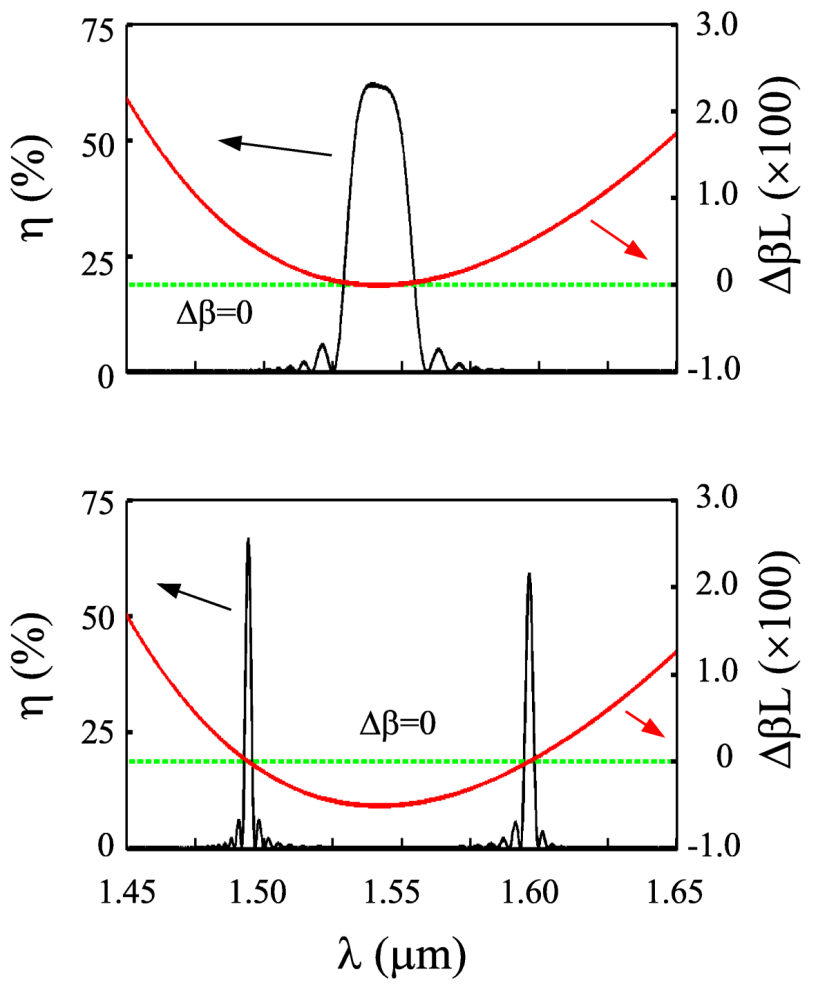

Fig. 4. SHG conversion efficiency (left vertical axes) and phase mismatch (right vertical axes) versus $\lambda_{F F}$ in a $1 \mathrm{~mm}$-long structure. Top: optimum $v$ and $w$ from Fig. 3. Bottom: $v$ was reduced by $\sim 5 \%$ with respect to the top panel. Crossings between the horizontal line and the detuning curve correspond to PM.

In conclusion, we have proposed and numerically investigated a simple structure for easily tunable and broad-band SHG. The scheme, suitable of straightforward extension to other parametric interactions, permits phase matching even in isotropic crystals, while preserving the nonlinear material response and a large overlap integral. The waveguide with a nano-void, well realizable with current technologies, allows extra tuning by, e.g., the infiltration of thermo- or electro-optic dielectrics or the use of microtransducers for real-time adjustment of the air-gap as well as direct modulation of the fields through the introduction of controlled mismatch (i.e., via cascading ${ }^{20}$ ). We anticipate a whole new family of ultra-compact doublers and mixers.

Acknowledgements. We thank the European Union (2004-512450 POISE) and the Italian Ministry for Research (PRIN 2005098337).

\section{References}

1. P. A. Franken, A. E. Hill, C. W. Peters and G. Weinreich, Phys. Rev. Lett. 7, 118 (1961).

2. J. A. Armstrong, N. Bloembergen, J. Ducuing and P. S. Pershan, Phys. Rev. 127, 1918 (1962).

3. R. W. Boyd, Nonlinear Optics (Academic Press, New York 2002)

4. M. M. Fejer, G. A. Magel, D. H. Jundt, R. L. Byer, IEEE J. Quantum Electron. 28, 2631 (1992).

5. Y. J. Ding, J. B. Khurgin, Opt. Lett. 21, 1445 (1996)

6. P. J. Vella, R. Normandin and G. I. Stegeman, Appl. Phys. Lett. 38, 759 (1981)

7. M. Ravaro, Y. Seurin, S. Ducci, G. Leo, V. Berger, A. DeRossi, G. Assanto, J. Appl. Phys. 98, 063103 (2005)

8. M. B. Raybaut, R. Hadar, Ph. Kupecek, Ph. Lemasson, E. Rosencher, Nature 432, 374 (2004).

9. C. D'Aguanno, M. Centini, M. Scalora, C. Sibilia, Y. Dumeige, P. Vidakovic, J. A. Levenson, M. J. Bloemer, C. M. Bowden, J. W. Haus, M. Bertolotti, Phys. Rev. E 64, 016609 (2001)

10. A. DiFalco, C. Conti, G. Assanto, Opt. Lett. 31, 250 (2006)

11. J. P. Van der Ziel, Appl. Phys. Lett. 26, 60 (1975)

12. A. Fiore, V. Berger, E. Rosencher, P. Bravetti, J. Nagle, Nature 391, 463 (1998)

13. K. Moutzouris, S. V. Rao, M. Ebrahimzadeh, A. DeRossi, M. Calligaro, V. Ortiz, V. Berger, Appl. Phys. Lett. 83, 620 (2003)

14. A. DeRossi, V. Berger, G. Leo and G. Assanto, IEEE J. Quantum Electron. 41, 1293 (2005)

15. J. D. Joannopoulos, P. R. Villeneuve, S. Fan, Photonic Crystals (Princeton Univ. Press, 1995)

16. Q. Xu, V. R. Almeida, R. R. Panepucci, and M. Lipson, Opt. Lett. 29, 1626 (2004)

17. M. A. Afromovitz, Solid State Commun. 15, 59 (1974)

18. S. Combrié, A. De Rossi, L. Morvan, S. Tonda, S. Cassette, D. Dolfi and A. Talneau, Electron. Lett. 42, 86 (2006)

19. M. V. Kotlyar, L. O'Faolain, R. Wilson, T. F. Krauss, J. Vac. Sci. Technol. B 22, 1788 (2004).

20. G. Assanto, G. Stegeman, M. Sheik-Bahae, E. VanStryland, IEEE J. Quantum Electron. 31, 673 (1995). 